\title{
Dose escalation study of bendamustine based conditioning regime (Bace-bendamustine, cytarabine, cyclophosphamide and etoposide) in patients with lymphoma undergoing autologous stem cell transplant
}

\begin{abstract}
Autologous Stem Cell Transplantation (ASCT) is the main stay of care in relapsed lymphomas including diffuse large B-cell lymphoma (DLBCL). There are different conditioning regimens which differ in efficacy and toxicity. Carmustine, etoposide, ARA-C, melphalan (BEAM), lomustine, etoposide, ARA-C, melphalan (LEAM) and lomustine, ARA-C, cyclophosphamide, etoposide (LACE) are commonly used condition in regimes. Therefore a less toxic regimen might improve the results in relapsed lymphoma patients.
\end{abstract}

Keywords: cell transplantation, carmustine, etoposide, toxic regimen
Volume 5 Issue 5 - 2017

\author{
Nidhi Dikshit,' Rajani Sinha,' Nitin Sood, ${ }^{2}$ \\ Anil Arabandi, ${ }^{3}$ Kasturi Sengupta,' Joydeep \\ Chakrabartty' \\ 'AMRI hospitals, India \\ ${ }^{2}$ Medanta Medi City, India \\ ${ }^{3}$ American Hospital, India
}

Correspondence: Nidhi Dikshit,AMRI hospitals, Kolkata, India, Email nidhidikshit17@gmail.com

Received: October 31, 2017 | Published: November 29, 2017
Abbreviations: ASCT, autologous stem cell transplantation; DLBCL, diffuse large B-cell lymphoma; LEAM, lomustine, etoposide, ara-C, melphalan; LACE, lomustine, ara-C, cyclophosphamide, etoposide; BeEAM, bendamustine, etoposide, cytarabine, melphalan; NHL, non hodgkin's lymphoma; HD, hodgkin's disease; IPI, international prognostic index; IPS, international prognostic score; HL, hodgkins lymphoma

\section{Introduction}

Autologous Stem Cell Transplantation (ASCT) is a standard of care in relapsed diffuse large B-cell lymphoma (DLBCL) and other lymphoproliferative disorders including relapsed Hodgkin's disease, mantle or T-cell lymphoma. Carmustine, etoposide, ARA-C, melphalan (BEAM) ${ }^{1}$ lomustine, etoposide, ARA-C, melphalan (LEAM) and lomustine, ARA-C, cyclophosphamide, etoposide (LACE) are commonly used condition in regimes. However, Carmustine and lomustine are associated with interstitial pneumonia ( 2 to 20\%) and increased risk of morbidity and mortality. Therefore a less toxic regimen might improve the results in relapsed lymphoma patients.

Bendamustine has already been shown to be a feasible part of a modified BEAM (bendamustine, etoposide, cytarabine, melphalan) ASCT condition in regimen for Non-Hodgkin's lymphoma with acceptable safety and promising efficacy. ${ }^{2}$ Bendamustine has a very different pharmacologic profile from alkylating agents. It combines the alkylating activity of the mustard group with the anti-metabolite activity of the purine analog structure. ${ }^{3}$

Two studies were carried out to determine the use of Bendamustine in lymphoma condition in regimen. In the first study, it was proposed that replacing Lomustine with Bendamustine would increase the efficacy and would be well tolerated in these patients needing an auto graft. ${ }^{4}$ The second study was a dose escalation study of Bendamustine, where a higher dose of Bendamustine $\left(300 \mathrm{mg} / \mathrm{m}^{2}\right)$ was used.

\section{Objective}

The objective of the first study was to assess the safety and efficacy of Bendamustine $\left(200 \mathrm{mg} / \mathrm{m}^{2}\right)$ based conditioning regimen for patients with lymphoma undergoing autologous stem cell transplant while the second study was carried out to determine the safety and efficacy of high dose Bendamustine $\left(300 \mathrm{mg} / \mathrm{m}^{2}\right)$ based conditioning regimen for patients with lymphoma undergoing autologous stem cell transplant.

\section{Materials and methods}

The first multi centric study was conducted between June 2012 and May 2014. Seventeen patients suffering from relapsed and/or refractory Non Hodgkin's Lymphoma (NHL) and Hodgkin's Disease (HD), previously treated with multiple combinations of chemotherapy were included in the study. The patients underwent peripheral blood stem cell collection after $3^{\text {rd }}$ cycle of salvage chemotherapy. Mobilization was done with G-CSF in thirteen patients and four patients required Plerixafor along with G-CSF. The conditioning regime used was R-BACE.

\section{R-BACE-regime in Study I consisted of :}

Rituximab- $375 \mathrm{mg} / \mathrm{m}^{2}$ on day- 6 , Bendamustine- $200 \mathrm{mg} / \mathrm{m}^{2}$ on day-6, Etoposide- $1000 \mathrm{mg} / \mathrm{m}^{2}$ on day-6, ARA-C- $2000 \mathrm{mg} / \mathrm{m}^{2}$ on day- 5 and day- 4 and Cyclophosphamide- $1,800 \mathrm{mg} / \mathrm{m}^{2}$ on day-3 to day-1.

The second study, conducted from June 2014 to May 2016 in multiple institutes included a total of 12 patients of relapsed and/or refractory Non Hodgkin's Lymphoma (NHL) and Hodgkins Disease 
(HD), previously treated with multiple combinations of chemotherapy. Here, this calated dose of Bendamustine $-300 \mathrm{mg} / \mathrm{m}^{2}$ was used and the results were compared with the previous study where the dose was $200 \mathrm{mg} / \mathrm{m}^{2}$. The conditioning regimes used in the second study were R-BACE/BACE.

\section{R-BACE-regime in Study 2 consisted of:}

Rituximab- $375 \mathrm{mg} / \mathrm{m}^{2}$ on day- 6 , Bendamustine $-300 \mathrm{mg} / \mathrm{m}^{2}$ on day $-6,-5$, Etoposide- $1000 \mathrm{mg} / \mathrm{m}^{2}$ on day-6, ARA-C- $2000 \mathrm{mg} / \mathrm{m}^{2}$ on day- 5 and day- 4 and Cyclophosphamide- $1,800 \mathrm{mg} / \mathrm{m}^{2}$ on day-3 to day- I.

In both the studies, autologous stem cells were infused on day 0 . Functional pulmonary tests and cardiac evaluation were done for all patients. Patients under went daily assessment of symptoms and physical examination during their hospital stay and weekly after discharge. Laboratory work-up was performed before transplantation, daily during the hospital stay, and weekly after discharge. Incidence and grade of treatment related toxicity were recorded according to CTCAE scale.

\section{Results}

The results of both the studies are summarized below

\section{Study I}

Out of seventeen patients who underwent autologous stem cell transplant, fourteen $(82.3 \%)$ patients were in complete remission and three $(17.7 \%)$ were in partial remission. The median number of lines of chemotherapy pre-transplant was 2 . None of the study patients developed more than grade 2 mucositis. The median number of CD34 positive cells infused was 3.84 million per $\mathrm{kg}$ body weight of the patient (Figure1). Median time taken for myeloid engraftment was 13days, and 19 days for platelet engraftment from the day of stem cell infusion (Figure 2). Fourteen (82.3\%) patients developed neutropenic sepsis during the engraftment period, which was managed adequately with broad spectrum IV antibiotics. Interestingly, eight patients (Table 1) developed MDR (multidrug resistant) Klebsiella infections either in blood or urine but these was managed with appropriate antibiotics. None of the patients showed any significant pulmonary toxicity. Mucositis was significantly less than that with melphalan containing regime. PET-CT scan today 100 and at Table 2 one year did not reveal any uptake and the patients are doing well, in complete remissions of arand continue to be on follow-up.

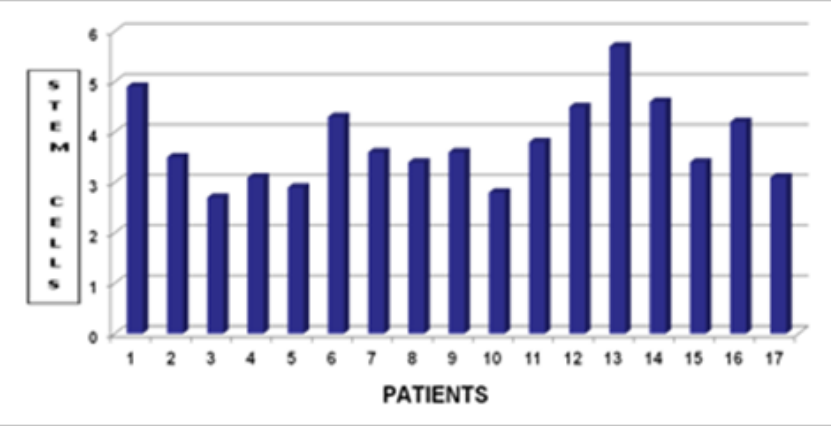

Figure I Stem cell (CD34+) dose.

\section{Study 2}

In this dose escalation study, out of the twelve patients who underwent autologous stem cell transplant, overall ten (83.3\%) patients were incomplete remission and two $(16.6 \%)$ were in partial remission. The median number of lines of chemotherapy pre-transplant was 2 . None of the study patients developed more than grade 2 mucositis. The median number of CD-34 positive cells infused was 4.54 million per $\mathrm{kg}$ body weight of the patient (Figure 3). Mean time taken for myeloid engraftment was 13 days, and 17 days for platelet engraftment from the day of stem cell infusion (Figure 4). There is no significant difference in the myeloid and platelet engraftment. Ten (83.3\%) patients developed neutropenic sepsis during the engraftment period, which was managed adequately with broad spectrum IV antibiotics. None of the patients showed any significant pulmonary toxicity. Mucositis was significantly less than melphalan containing regime, and also (Tables $3 \& 4$ ) comparable with previous dose of Bendamustine $\left(200 \mathrm{mg} / \mathrm{m}^{2}\right)$. One patient with a Grey Zone Lymphoma has relapsed 4months after his transplant. But the remaining 11 continue to be in remission.

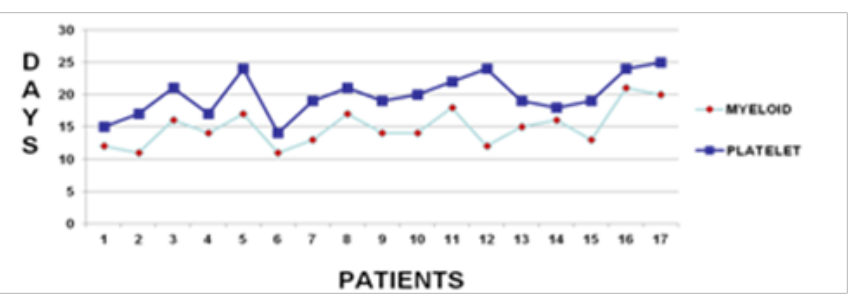

Figure 2 Myeloid and patient engraftment.

Table I Patient and disease characteristics

\begin{tabular}{ll}
\hline Characteristics & Patients N-I 7 \\
\hline Age & \\
Median (Range) & $49(39-56)$ \\
Gender & I2-May \\
Male/Female & \\
Diagnosis & 12 \\
DLBCL & 2 \\
MCL & 3 \\
HL & \\
ECOG Performance Status & 11 \\
0-I & 6 \\
$\geq 2$ & $01-A p r$ \\
International prognostic index (IPI) For NHL & 3 \\
International prognostic score (IPS) for Hodgkins & $03-A p r$ \\
disease & 17.8 (5-29) \\
Median Time: Diagnosis to transplant (months) & \\
Pre Transplant Disease Status & \\
CR & \\
PR & \\
\hline
\end{tabular}

DLBCL, diffuse large B-cell lymphoma, MCL, mante cell lymphoma, $\mathrm{HL}$, hodgkins lymphoma

Table 2 Common hematologic adverse effects

\begin{tabular}{llll}
\hline Adverse effect & All grades No. & Grade No. 3 & Grade No. 4 \\
\hline Anemia & 07 & 05 & 02 \\
Neutropenia & 15 & 09 & 06 \\
Thrombocytopenia & 16 & 07 & 09 \\
Febrile neutropenia & 08 & 07 & 01 \\
\hline
\end{tabular}




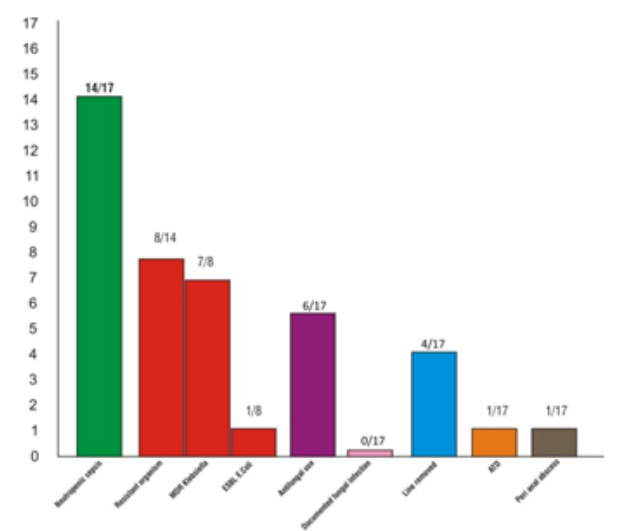

Figure 3 Infection data-study I.

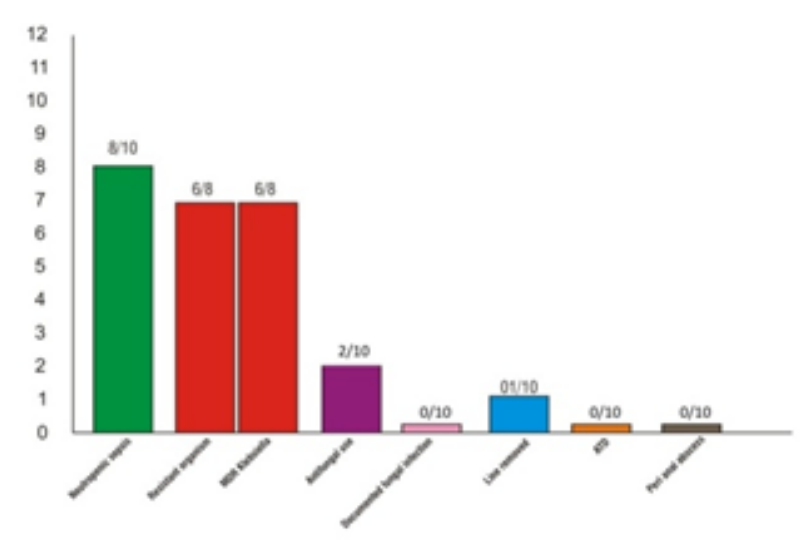

Figure 4 Infection data-study 2.

Table 3 Patient and disease characteristics

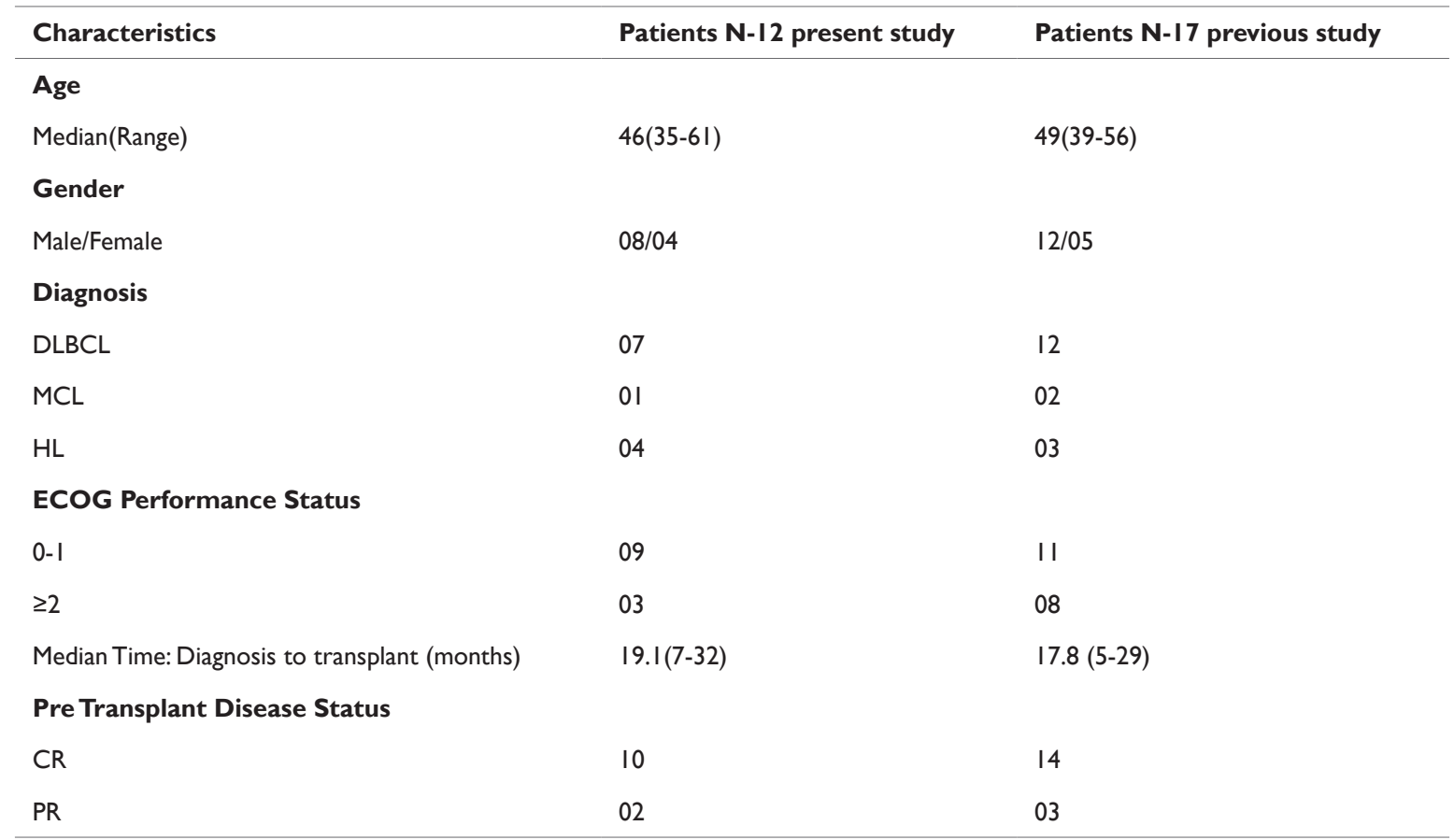

DLBCL, diffuse large B-cell lymphoma, MC, mante cell lymphoma, HL, hodgkins lymphoma

Table 4 Common hematologic adverse effects

\begin{tabular}{lllllc}
\hline \multirow{2}{*}{ Adverse effect } & All Grades No. & & Grade No. 3 & \multicolumn{2}{c}{ Grade No. 4 } \\
\cline { 2 - 6 } & Present Study & Previous study & Present study & Previous study & Present study \\
\hline Anemia & 08 & 07 & 06 & 05 & 02 \\
Neutropenia & 11 & 15 & 10 & 09 & 01 \\
Thrombocytopenia & 10 & 16 & 09 & 07 & 01 \\
Febrile neutropenia & 09 & 08 & 07 & 07 & 09 \\
\hline
\end{tabular}

\section{Discussion}

ASCT condition in regimes for Lymphoma generally includes BEAM, LACE or LEAM. The practice varies between institutes and there have been no randomized controlled trials to show which one is superior. Bendamustine containing regimes are now being used
(BEEAM) with similar or greater efficacy and less toxicity. ${ }^{5}$ Toxicities of BCNU-containing regimens, such as BEAM area cause for concern because of high incidence of interstitial pneumonitis. Patients who receive carmustine as part of their preparative regimen for stem cell transplantation offer a longer window in which the development of pulmonary complications can occur. In this setting, the incidence of 
interstitial pneumonitis and idiopathic pneumonia has been reported to be $2 \%-23 \%$. Hence, the identification of safe and effective conditioning regime to maximize ASCT response is still a challenge. Bendamustine has proven clinical activity in resistant/relapsed B-cell malignancies, such as indolent lymphomas and chronic lymphocytic leukemia. It has demonstrated the greater efficacy in combination with etoposide, cytarabine and melphalan with respect to carmustine.

In Study 1 , the safety and efficacy of a new high dose chemotherapy regimen with Bendamustine, Cytarabine, Cyclophosphamide and Etoposide (BACE) prior to autologous stem cell rescue in 17patients with relapsed/refractory lymphoma was demonstrated following the use of Bendamustine at $200 \mathrm{mg} / \mathrm{m}^{2}$. Transplant-related mortality was $0 \%$. The cumulative incidence of infectious complications was approximately $50 \%$, without any serious adverse events (grades 3-4). Furthermore, this regimen showed significant anti-lymphoma activity, with $100 \%$ of patients being incomplete remission one year after transplant. LACE (lomustine, cytarabine (Ara-C), cyclophosphamide, etoposide) and CCNU substitution for BCNU in the $\mathrm{CBV}$ (cyclophosphamide, BCNU, etoposide) regimen are some lomustine based regimens that have shown comparable results. In this multi-centre study, the authors examined the toxicities and efficacy of the LACE regimen as a historical control vis-à-vis BACE which was used in these patients. No significant difference was observed in the peritransplant out comes in terms of engraftment, febrile complications, intravenous antibiotic use and duration of hospitalization. However, grade $3 / 4$ mucositis $(67 \%)$ and diarrhea $(45 \%)$ have been found in LACE based regime which is not noted in the present study. Rate of pulmonary toxicity is also comparatively high in LACE based regime but none occurred in this patient population.

In Study 2, the results were compared with those of Study 1 (Figure 5). Here, the dose of Bendamustine was escalated to $300 \mathrm{mg} /$ $\mathrm{m}^{2}$. So far, 12patients have been studied and the authors did not notice any increased complications and adverse events in comparison to previous study with Bendamustine dose of $200 \mathrm{mg} / \mathrm{m}^{2}$. As in the first study, transplant- related mortality was $0 \%$ and with significant antilymphoma activity and $100 \%$ patients being incomplete remission at least 8 months after transplant. After assessing the toxicity and efficacy of the BACE (Bendamustine $@ 300 \mathrm{mg} / \mathrm{m}^{2}$ ) regimen, no significant difference in the peritransplant outcomes in terms of engraftment, febrile complications, intravenous antibiotic use, and duration of hospitalization was noted between the two Bendamustine dose regimens.

\section{Conclusion}

In conclusion, BACE as conditioning regime followed by ASCT is feasible and effective in patients with Non-Hodgkin's lymphoma and Hodgkin's disease. ${ }^{6}$ Toxicity of this chemotherapy is acceptable and definitely lower than that observed with the standard BEAM or LACE regime. But the incidence of MDR bacterial infections is very high in India and urgent measures are needed to address this issue. There was no Grade3-4 non haematological toxicity and the maximum mucositis ${ }^{7}$ encountered was Grade2. Thus, the use of Bendamustine in lymphoma conditioning regimen can be recommended on the basis of its high anti-lymphoma activity with a lower toxicity. Moreover, Bendamustine is an effective drug when given with other combination chemotherapy as a conditioning regime and is well to le rated even at a higher dose of $300 \mathrm{mg} / \mathrm{m}^{2}$.

\section{Acknowledgements}

None.

\section{Conflict of interest}

The author declares no conflict of interest.

\section{References}

1. Visani G, Stefani PM, Capria S, et al. Bendamustine, etoposide, cytarabine, melphalan, and autologous stem cell rescue produce a $72 \%$ 3-year PFS in resistant lymphoma. Blood. 2014;124(19):3029-3031.

2. Isidori A, Patriarca F. High dose therapy with bendamustine, etoposide, ara-C, melphalan (BeEAM) prior to autologous stem cell transplant is safe and effective in aggressive B-cell non Hodgkin lymphoma: an Italian phaseII, multicenter study. Haematologica. 2014;99:s27.

3. Visani G, Malerba L, Stefani PM, et al. BeEAM (bendamustine, etoposide, cytarabine, melphalan) before autologous stem cell transplantation is safe and effective for resistant/relapsed lymphoma patients; Blood. 2011;118(12):3419-3425.

4. Kumar N, Joshi A, Shah M, et al. Comparison of toxicity of BEAM vs. LACE regimen in patients of lymphoma [abstract]. In: $35^{\text {th }}$ Annual Meeting of the European Group for Blood and Marrow Transplantation. Göteborg, Sweden; 2009.

5. LeoniL M, Bailey B, Reifert J, et al. Bendamustine displays a distinct pattern of cytotoxicity and unique mechanistic features compared with other alkylating agents. Clin Cancer Res. 2008;14(1):309-317.

6. Appelbaum FR. Hematopoietic cell transplantation for nonHodgkin's lymphoma: yesterday, today, and tomorrow. J Clin Oncol. 2008;26(18):2927-2929.

7. Chakrabartty J, Jain N. Biology of blood and marrow transplantation. 2016;3:S121-S122. 